\title{
Correction to: Adaptive dynamics of saturated polymorphisms
}

\author{
Éva Kisdi ${ }^{1}$ - Stefan A. H. Geritz ${ }^{1}$ \\ Published online: 10 August 2019 \\ ๑) Springer-Verlag GmbH Germany, part of Springer Nature 2019
}

\section{Correction to: J. Math. Biol. (2016) 72:1039-1079 https://doi.org/10.1007/s00285-015-0948-2}

In the original publication, Proposition 4 is mistaken. The proposition considers models with two environmental feedback variables, $E_{1}$ and $E_{2}$, and two resident strategies with trait values $x_{1}$ and $x_{2}$. The original proposition claimed that if the invasion fitness $r\left(x, E_{1}, E_{2}\right)$ is uniformly monotonic in $E_{i}(i=1$ or 2$)$, then the equilibrium value of this same feedback as a function of the trait values, $\hat{E}_{i}\left(x_{1}, x_{2}\right)$, has a saddle point at a generic evolutionarily stable singularity. In fact, it is not $\hat{E}_{i}\left(x_{1}, x_{2}\right)$, but the other feedback that has a saddle point under the conditions stated in Proposition 4 of the original publication. The corrected proposition and its proof appear below. The error does not affect the rest of the article.

In most models that we encounter, the invasion fitness is uniformly monotonic in all feedback variables (this also pertains to the example presented in Section 2 of the original publication). In this case, both feedbacks have a saddle point and the correction of the proposition has no practical consequence; yet the proof needs to be corrected.

Proposition 4 Suppose there are two environmental feedback variables and two coexisting resident strategies $(n=2)$. If the invasion fitness is uniformly monotonic in the environmental feedback variable $E_{1}$, then $\hat{E}_{2}\left(x_{1}, x_{2}\right)$ has a saddle point at a generic evolutionarily stable dimorphic singularity, and vice versa, if the invasion fitness is uniformly monotonic in $E_{2}$, then $\hat{E}_{1}\left(x_{1}, x_{2}\right)$ has a saddle point at a generic evolutionarily stable dimorphic singularity. Specifically, the environmental feedback at the saddle is maximized as a function of one trait value and minimized as a function of the other.

The original article can be found online at https://doi.org/10.1007/s00285-015-0948-2.

Éva Kisdi

eva.kisdi@helsinki.fi

1 Department of Mathematics and Statistics, University of Helsinki, PO Box 68, 00014 Helsinki, Finland 
Proof For $n=2$, equation (16) of the original article reads

$$
\mathbf{A}\left[\begin{array}{c}
\frac{\partial^{2} \hat{E}_{1}}{\partial x_{j} \partial x_{k}} \\
\frac{\partial^{2} \hat{E}_{2}}{\partial x_{j} \partial x_{k}}
\end{array}\right]=\left\{\begin{array}{cl}
-\partial_{11} r\left(x_{j}, \hat{E}_{1}, \hat{E}_{2}\right) \mathbf{e}_{j} & \text { if } k=j \\
\mathbf{0} & \text { otherwise }
\end{array}\right.
$$

where $j, k \in\{1,2\}$ and the matrix $\mathbf{A}$ has elements $A_{i j}=\partial_{j+1} r\left(x_{i}, \hat{E}_{1}, \hat{E}_{2}\right)$. In particular, with $k=j$, we have

$$
\mathbf{A}\left[\begin{array}{c}
\frac{\partial^{2} \hat{E}_{1}}{\partial x_{1}^{2}} \\
\frac{\partial^{2} \hat{E}_{2}}{\partial x_{1}^{2}}
\end{array}\right]=\left[\begin{array}{c}
-\partial_{11} r\left(x_{1}, \hat{E}_{1}, \hat{E}_{2}\right) \\
0
\end{array}\right]
$$

and

$$
\mathbf{A}\left[\begin{array}{c}
\frac{\partial^{2} \hat{E}_{1}}{\partial x_{2}^{2}} \\
\frac{\partial^{2} E_{2}}{\partial x_{2}^{2}}
\end{array}\right]=\left[\begin{array}{c}
0 \\
-\partial_{11} r\left(x_{2}, \hat{E}_{1}, \hat{E}_{2}\right)
\end{array}\right]
$$

for $j=1$ and for $j=2$, respectively. Using Cramer's rule, we thus obtain

$$
\begin{aligned}
\frac{\partial^{2} \hat{E}_{1}}{\partial x_{1}^{2}} & =\frac{-\partial_{11} r\left(x_{1}, \hat{E}_{1}, \hat{E}_{2}\right) \cdot \partial_{3} r\left(x_{2}, \hat{E}_{1}, \hat{E}_{2}\right)}{\operatorname{det} \mathbf{A}} \\
\frac{\partial^{2} \hat{E}_{1}}{\partial x_{2}^{2}} & =\frac{\partial_{11} r\left(x_{2}, \hat{E}_{1}, \hat{E}_{2}\right) \cdot \partial_{3} r\left(x_{1}, \hat{E}_{1}, \hat{E}_{2}\right)}{\operatorname{det} \mathbf{A}}
\end{aligned}
$$

and

$$
\begin{aligned}
\frac{\partial^{2} \hat{E}_{2}}{\partial x_{1}^{2}} & =\frac{\partial_{11} r\left(x_{1}, \hat{E}_{1}, \hat{E}_{2}\right) \cdot \partial_{2} r\left(x_{2}, \hat{E}_{1}, \hat{E}_{2}\right)}{\operatorname{det} \mathbf{A}} \\
\frac{\partial^{2} \hat{E}_{2}}{\partial x_{2}^{2}} & =\frac{-\partial_{11} r\left(x_{2}, \hat{E}_{1}, \hat{E}_{2}\right) \cdot \partial_{2} r\left(x_{1}, \hat{E}_{1}, \hat{E}_{2}\right)}{\operatorname{det} \mathbf{A}}
\end{aligned}
$$

The second derivatives $\partial_{11} r\left(x_{1}, \hat{E}_{1}, \hat{E}_{2}\right)$ and $\partial_{11} r\left(x_{2}, \hat{E}_{1}, \hat{E}_{2}\right)$ are both negative at a generic evolutionarily stable singularity. If the invasion fitness is uniformly monotonic in $E_{2}$, then the factors $\partial_{3} r\left(x_{2}, \hat{E}_{1}, \hat{E}_{2}\right)$ and $\partial_{3} r\left(x_{1}, \hat{E}_{1}, \hat{E}_{2}\right)$ in Eqs. (1a) and (1b) have the same sign. Due to the minus sign that appears in (1a) but not in (1b), $\frac{\partial^{2} \hat{E}_{1}}{\partial x_{1}^{2}}$ and $\frac{\partial^{2} \hat{E}_{1}}{\partial x_{2}^{2}}$ have opposite signs. Together with Proposition 2 of the original article, this shows that $\hat{E}_{1}\left(x_{1}, x_{2}\right)$ is maximized as a function of one trait value and minimized as a function of the other, i.e., it has a saddle point at a generic evolutionarily stable dimorphic singularity. Similarly, if the invasion fitness is uniformly monotonic in $E_{1}$, then the factors $\partial_{2} r\left(x_{2}, \hat{E}_{1}, \hat{E}_{2}\right)$ and $\partial_{2} r\left(x_{1}, \hat{E}_{1}, \hat{E}_{2}\right)$ in Eqs. (2a) and (2b) have the same sign, 
so that $\frac{\partial^{2} \hat{E}_{2}}{\partial x_{1}^{2}}$ and $\frac{\partial^{2} \hat{E}_{2}}{\partial x_{2}^{2}}$ have opposite signs; $\hat{E}_{2}\left(x_{1}, x_{2}\right)$ is therefore maximized as a function of one trait value and minimized as a function of the other.

Publisher's Note Springer Nature remains neutral with regard to jurisdictional claims in published maps and institutional affiliations. 